\title{
TIA-1 Positive Tumor-Infiltrating Lymphocytes in Nevi and Melanomas
}

\author{
Stephen Lyle, M.D., Ph.D., Kevin E. Salhany, M.D., ${ }^{*}$ David E. Elder, M.B.Ch.B., F.R.C.P.A. \\ Department of Pathology and Laboratory Medicine, University of Pennsylvania Medical Center, \\ Philadelphia, Pennsylvania
}

Tumor-infiltrating lymphocytes (TIL) have been shown to be an independent prognostic factor in melanomas. To better characterize the host immune response, we have classified TIL by their immunoreactivity against lymphoid markers in formalin-fixed, paraffin-embedded tissue. Monoclonal antibodies to leukocyte common antigen (LCA) and TIA-1 (a granule-associated protein of cytotoxic T cells and NK cells) were used to immunostain a series of benign nevi, nontumorigenic radial growth phase, and tumorigenic vertical growth phase melanomas and metastases. Among nine nevi, few $\mathrm{LCA}^{+}$TIL were found, among which rare cells were positive for TIA-1 (mean, 2.0). Five nontumorigenic radial growth phase melanomas also had few total TIL and rare TIA-1 ${ }^{+}$TIL (mean, 3.4); the nontumorigenic radial growth phase component of seven tumorigenic vertical growth phase melanomas had higher numbers of TIA-1 ${ }^{+}$TIL (mean, 11). Twelve cases of tumorigenic vertical growth phase melanoma showed a variable but significantly greater number of both LCA $^{+}$TIL and TIA-1 ${ }^{+}$TIL (mean, 30.6). Nine cases of metastatic melanoma had a wide range of variation in LCA as well as in TIA-1 ${ }^{+}$TIL (mean, 46). Although the mean total number of TIA-1 ${ }^{+}$TIL increased from nontumorigenic radial growth phase to tumorigenic vertical growth phase to metastases, TIA- $1^{+}$as a percentage of TIL declined across these categories of tumor progression $(42 \%, 31 \%$, and $26 \%$, respectively). Our results show that these attributes of TIA- ${ }^{+}$ TIL, both increasing total number but decreasing percentage, appear to be a marker of tumor progression of malignant melanomas. In addition,

Copyright (C) 2000 by The United States and Canadian Academy of Pathology, Inc.

VOL. 13, NO. 1, P. 52, 2000 Printed in the U.S.A.

Date of acceptance: June 17, 1999.

* Deceased.

This work supported by CA25874 and CA75434 from the National Cancer Institute.

Address reprint requests to: David E. Elder, M.B., Ch.B., Hospital of the University of Pennsylvania, Department of Pathology and Laboratory Medicine, 3400 Spruce Street, 6 Founders Pavilion, Philadelphia, PA 19104; fax: 215-349-5910. there was significant variability in the number of TIA- $1^{+}$TIL among advanced melanomas, raising the possibility that an assessment of TIA-1 ${ }^{+}$TIL may prove a useful prognostic tool for the evaluation of primary melanomas.

KEY WORDS: Melanoma, TIA-1, Tumor-infiltrating lymphocytes.

Mod Pathol 2000;13(1):52-55

Primary cutaneous malignant melanomas frequently incite a cellular immune response characterized by lymphocytes that surround and may infiltrate the tumor. Tumor-infiltrating lymphocytes (TIL), defined as lymphocytes that infiltrate into the tumor and are in direct contact with tumor cells, have been shown to be an independent prognostic factor in primary tumorigenic vertical growth phase (VGP) melanomas (1-3). TIL have been classified as brisk, non-brisk, and absent in the prognostic model. TIL have also been shown to be prognostically important in predicting disease-free survival inpatients with lymph node metastases (4). These models have used the pattern of lymphocytic response including all lymphocytes identified morphologically by hematoxylin and eosin staining present in the infiltrate. However, the detailed phenotypic characterization of the lymphoid infiltrate in tissue sections of benign and malignant melanocytic lesions has not been reported, and it is not known whether all TIL are capable of tumor cell lysis.

Cytotoxic T-lymphocytes (CTLs) are the effector cells with the ability to recognize and induce target cell cytolysis (5). The activated cytolytic lymphocytes can mediate cell lysis by releasing cytoplasmic granules which contain perforin, granzymes, and TIA-1 $(6,7)$. On release from cytoplasmic granules, perforin inserts into the target cell cytoplasmic membrane and polymerizes to form membrane pores. Pore formation can cause direct cell lysis through osmotic forces and the passage of small molecules such as calcium. In addition, the pores may also allow for the influx of proteins into the 
target cell. Granzymes are serine proteases that are involved in the pathway of DNA breakdown. They act inside the target cell to cleave substrates, eventually leading to apoptosis. TIA-1 is an intracellular protein of cytotoxic lymphocytes that is localized within the cytoplasmic granules containing serine proteases. It is a $15 \mathrm{kD}$ granule membraneassociated protein that can induce apoptotic cell death when introduced into permeablized cells (8). Monoclonal antibodies against TIA-1 define a subpopulation of $\mathrm{CD}^{+}$T-cells and NK cells that have cytotoxic activity (7-9). Immunostaining using these antibodies produces a granular, cytoplasmic staining pattern in CTLs and NK cells and can be done in paraffin-embedded tissue. TIA-1 specific antibodies could be a useful tool to study the immune response to malignancies in which there are TIL.

Because melanoma is a classic example of a tumor in which the host response plays a major role in the determination of prognosis $(1-4,10,11)$, additional variables such as the classification of TIL may improve the specificity and predictive value of current prognostic models. Therefore, we report the characterization of TIL using the cytotoxic T-cell marker, TIA-1, in a variety of melanocytic tumors from benign nevi to metastatic melanoma.

\section{MATERIALS AND METHODS}

Formalin-fixed, paraffin embedded archival tissue from melanocytic lesions including benign nevi (dermal, compound, and dysplastic types), nontumorigenic radial growth phase (RGP) and VGP primary melanomas and metastases was immunostained with monoclonal antibodies to LCA, (DAKO), and TIA-1 (Coulter) using the AvidinBiotin Complex method. None of the lesions showed any evidence of irritation, previous biopsy, or other cause of inflammation on H\&E stain. All tissue samples were steamed for 15 minutes before incubation with the primary antibody. LCA was used at a dilution of 1:100 and TIA-1 at 1:1000. TIL, defined as lymphocytes in direct contact with tumor cells, were quantitated by counting positively stained cells within a $0.25 \times 0.25 \mathrm{~mm}$ grid $(10 \times$ eyepiece with $40 \times$ objective) in the area of highest concentration within the tumor. The data were analyzed using the SPSS statistics program (SPSS, Chicago, IL).

\section{RESULTS}

TIL were counted and the mean value was calculated for each category of lesion (Table 1). Among nine nevi, few $\mathrm{LCA}^{+}$TIL were found, among which rare cells were positive for TIA-1 (Fig. 1). Five RGP melanomas also had few total TIL and rare TIA- $1^{+}$ TIL (mean, 3); the RGP component of seven VGP melanomas had higher numbers of TIA- $1^{+}$TIL (mean, 11), (Fig. 2). Twelve cases of VGP melanoma showed a fourfold variation in $\mathrm{LCA}^{+}$TIL and a roughly 10 -fold variation in TIA- ${ }^{+}$TIL (Fig. 3). Nine cases of metastatic melanoma had a wide range of variation in $\mathrm{LCA}^{+}$as well as TIA-1 ${ }^{+}$TIL (Fig. 4). The TIA- $1^{+}$response as a percentage of total TIL declined from RGP (42\%) to VGP (31\%) to metastases (26\%).

Several of these differences were statistically significant. TIA- $1^{+}$cells in RGP melanomas were higher than dermal nevi $(P<.04) . \mathrm{LCA}^{+}$cells were higher in VGP melanomas than both dermal nevi $(P<.01)$ and RGP melanomas $(P<.01)$. Similarly, TIA- $1^{+}$cells were higher in VGP melanomas than both dermal nevi $(P<.01)$ and RGP melanomas $(P=.02)$. The difference between VGP and metastatic melanoma was not statistically significant.

TABLE 1. Immunoreactivity of Tumor-Infiltrating Lymphocytes

\begin{tabular}{|c|c|c|c|c|c|c|c|c|}
\hline & \multicolumn{2}{|c|}{ Nevi $(n=9)$} & \multicolumn{2}{|c|}{ RGP Melanoma $(n=12)$} & \multicolumn{2}{|c|}{ VGP Melanoma $(n=12)$} & \multicolumn{2}{|c|}{ Metastases $(n=9)$} \\
\hline & $\mathrm{LCA}^{+}$ & $\mathrm{TIA}^{+}(\%)$ & $\mathrm{LCA}^{+}$ & $\mathrm{TIA}^{+}(\%)$ & $\mathrm{LCA}^{+}$ & TIA $^{+}$ & $\mathrm{LCA}^{+}$ & $\mathrm{TIA}^{+}(\%)$ \\
\hline & 18 & $0(0)$ & 11 & $8(72)$ & 53 & $19(36)$ & 106 & $14(14)$ \\
\hline & 6 & $6(100)$ & 2 & $0(0)$ & 98 & $14(14)$ & 393 & $79(20)$ \\
\hline & 11 & $2(20)$ & 8 & $1(13)$ & 226 & $112(50)$ & 170 & $48(28)$ \\
\hline & 0 & $0(0)$ & 13 & $6(46)$ & 172 & 18 (10) & 337 & $61(18)$ \\
\hline & 16 & $2(13)$ & 14 & $6(43)$ & 69 & 45 (65) & 99 & 78 (79) \\
\hline & 0 & $0(0)$ & 16 & $2(13)$ & 49 & $26(53)$ & 235 & $17(7)$ \\
\hline & 26 & $7(27)$ & 57 & $30(52)$ & 119 & $9(8)$ & 152 & 59 (39) \\
\hline & 0 & $0(0)$ & 28 & $14(50)$ & 40 & $14(35)$ & 11 & 59 (55) \\
\hline & 2 & $1(50)$ & 30 & $1(3)$ & 119 & 38 (32) & 63 & $6(81)$ \\
\hline & & & 18 & $8(44)$ & 79 & $35(44)$ & & \\
\hline & & & 18 & $10(56)$ & 59 & 9 (15) & & \\
\hline & & & 14 & $5(36)$ & 110 & $28(25)$ & & \\
\hline Mean [SE] & $9[3]$ & $\begin{array}{l}2[1] \\
(22 \%)\end{array}$ & $19[4]$ & 8 [2] (42\%) & 99 [16] & $31[8](31 \%)$ & $174[42]$ & $46[9] \quad(26 \%)$ \\
\hline
\end{tabular}

RGP, radial growth phase; VGP, vertical growth phase. 


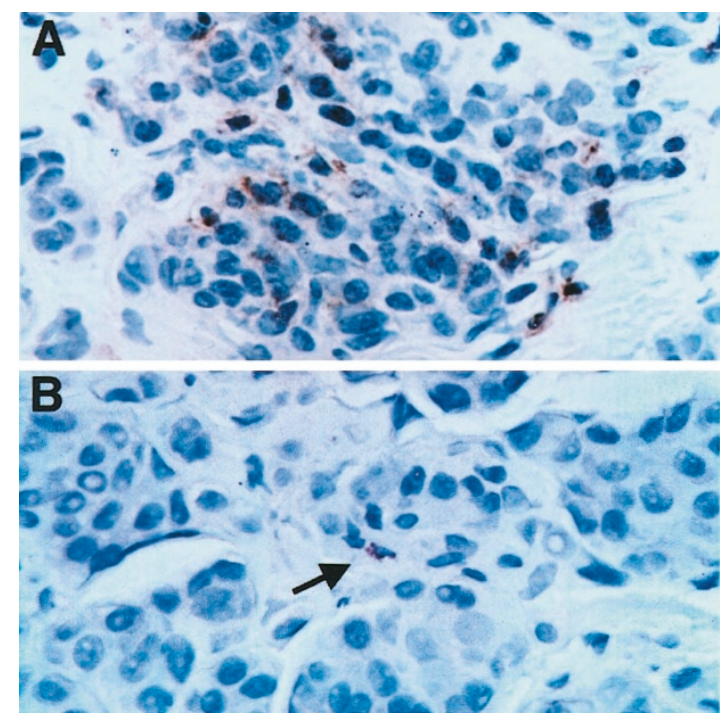

FIGURE 1. Dermal nevus with tumor-infiltrating lymphocytes stained for LCA (A) and TIA-1 (B). Although LCA ${ }^{+}$tumor-infiltrating lymphocytes can readily be found in dermal nevi, only rare isolated TIA- $1^{+}$tumorinfiltrating lymphocytes are seen (original magnification, $400 \times$ ).

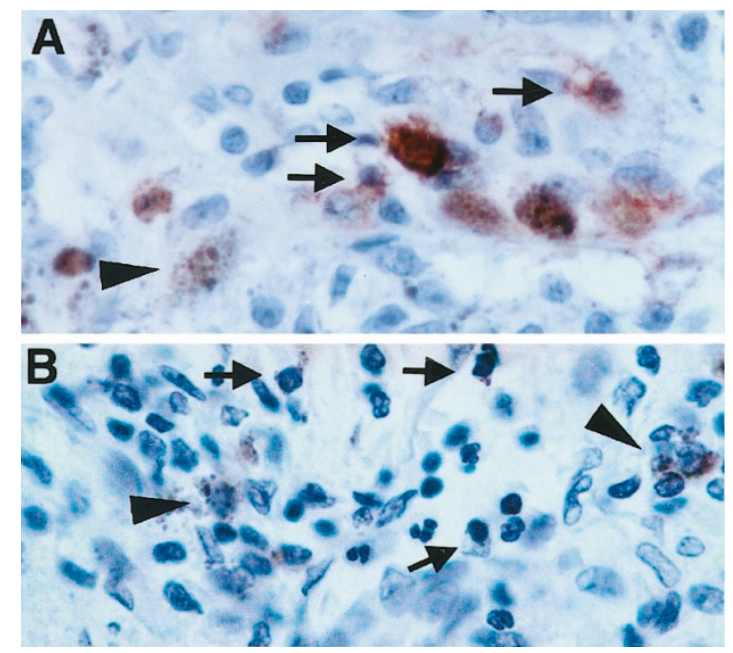

FIGURE 2. Radial growth phase melanoma with tumor-infiltrating lymphocytes stained for LCA (A) and TIA-1 (B). LCA ${ }^{+}$tumor-infiltrating lymphocytes with brown membrane staining (arrows) are distinguished from melanophages with coarse, granular pigment (arrowheads). TIA$1^{+}$tumor-infiltrating lymphocytes (arrows) can be distinguished from melanophages (arrowheads) by morphology (original magnification, $400 \times)$.

\section{DISCUSSION}

The current prognostic model for primary melanoma includes variables of both the neoplasm and the host response (for review, see 11). The presence of TIL, along with mitotic count, is one of the most powerful predictors of survival in VGP melanomas. However, a definitive immunohistochemical characterization of TIL using lymphoid markers in tissue sections of melanoma has not been done. It is not known whether the number of effector cells, capable of cytolysis, correlates with the infiltrate seen on routine H\&E stain. Our results show that TIL can easily be classified by their immunoreactivity against lymphoid

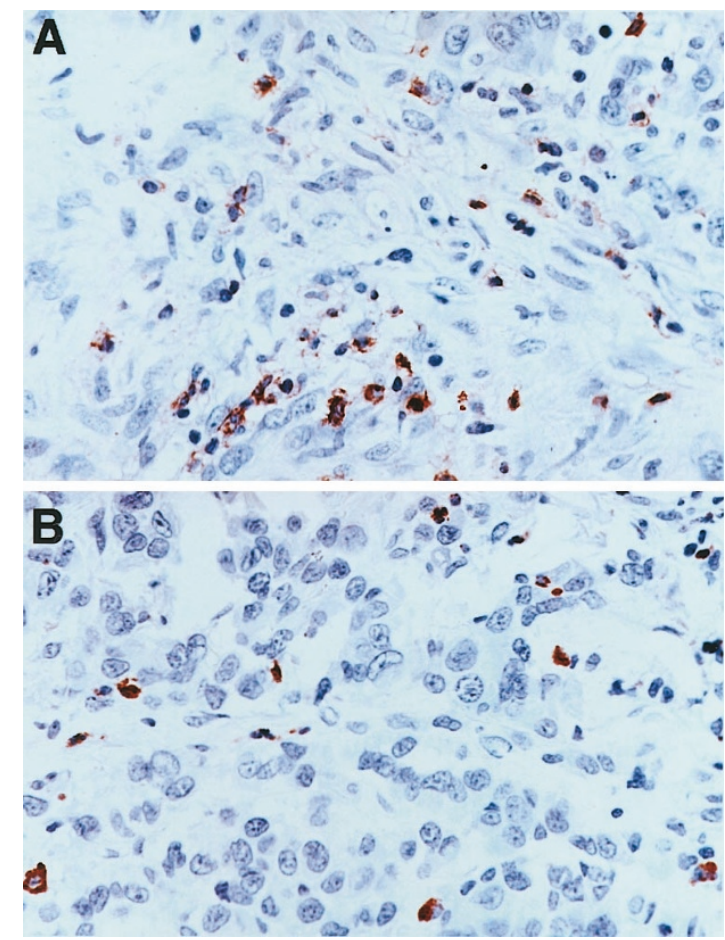

FIGURE 3. Vertical growth phase melanoma with tumor-infiltrating lymphocytes stained for LCA (A) and TIA-1 (B). Tumor nodules have focal areas with numerous $\mathrm{LCA}^{+}$tumor-infiltrating lymphocytes and variable numbers of TIA- $1^{+}$tumor-infiltrating lymphocytes (original magnification, $400 \times$ ).

markers in paraffin-embedded tissue. In addition, the total number of TIA- ${ }^{+}$TIL appears to be a marker of tumor progression of malignant melanomas. They are absent or rare in benign melanocytic lesions and radial growth phase melanomas, whereas vertical growth phase melanomas and metastases have higher numbers of these cytotoxic effector cells. $\mathrm{Cu}$ riously, although the total number of TIA- $1^{+}$TIL increases from RGP to metastatic melanomas, the percentage of TIA- $1^{+}$TIL actually decreases with tumor progression. This finding suggests that tumors that progress to VGP and metastatic melanoma have a less efficient TIL response.

Previous studies have shown that TIL in VGP and metastatic melanomas correlate with survival (1-4). In this study, we show that among the VGP and metastatic melanomas, there is a wide range in the number of TIA- $1^{+}$TIL. Although the cumulative data show a general correlation between the total TIL identified by LCA positivity and the number of TIA- $1^{+}$TIL, the two values occasionally diverge within individual cases. For instance, the percentage of TIA- $1^{+}$TIL in metastatic lesions ranges from $15 \%$ to $\sim 80 \%$. This heterogeneity in the host response suggests that the quantitation of cytotoxic T-cells identified by TIA- 1 immunoreactivity may segregate tumors based on the TIL's capability to effect target cell cytolysis. An infiltrate composed of more CTLs may signify a more effective host response and, thus, it may prove more useful in the 


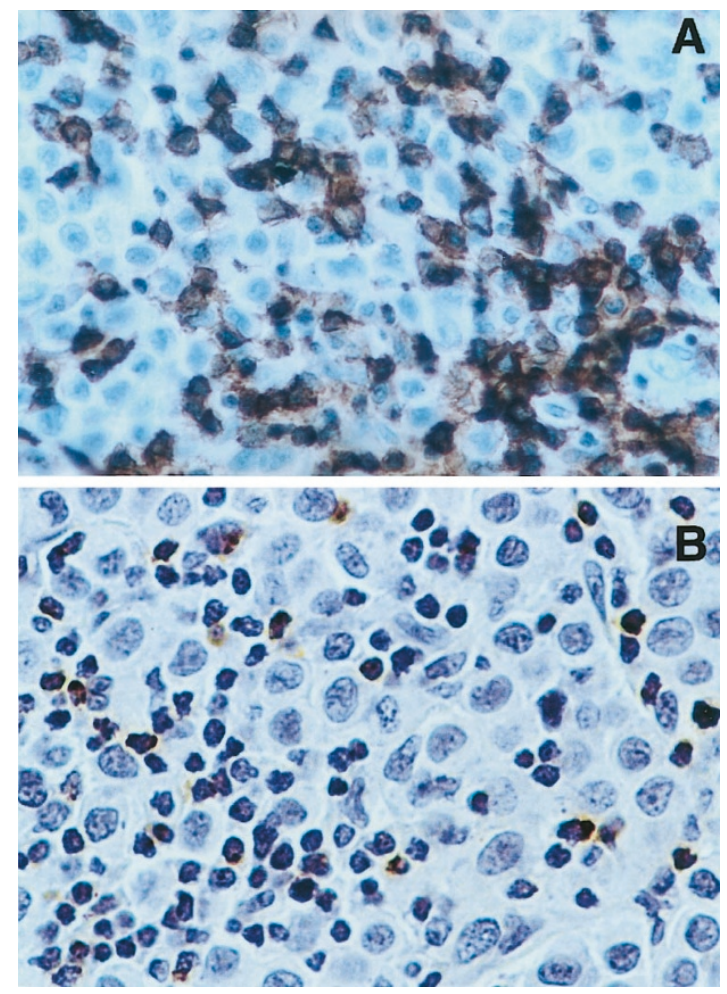

FIGURE 4. Dermal metastasis with tumor-infiltrating lymphocytes stained for LCA (A) and TIA-1 (B). Tumor nodules have foci with numerous $\mathrm{LCA}^{+}$tumor-infiltrating lymphocytes, many of which are TIA-1 positive (original magnification, $400 \times$ ).

prognostic model to determine the number of TIA-1 positive TIL in VGP melanomas. Survival was not addressed in the current study; future investigations will look at melanomas with 10 -year follow-up to determine the significance of TIA- $1^{+}$ TIL as a predictive factor.

TIL reinfusion, as well as the use of interleukin-2 to stimulate the antitumor immune response through sensitization of CTLs, has shown promise as a potential adjuvant therapy for melanoma (1217). The use of TIA-1 immunohistochemistry to identify CTLs may also help to stratify patients who would benefit from interleukin-2 or TIL infusion therapy. Patients with more TIA- $1^{+}$TIL may effect a better response to sensitization, or patients with less TIA- $1^{+}$TIL may recruit a greater number of TIL with stimulation. Correlation of the TIA-1 response with clinical outcome in melanoma could provide useful information to incorporate in future trials evaluating the response of interleukin-2, TIL infusion or other treatment modalities.

Acknowledgments: We would like to thank Shelley Roberts of the Dept. of Pathology and Lab Medicine at the University of Pennsylvania for her technical assistance.

\section{REFERENCES}

1. Clark WH, Elder DE, Guerry DPIV, Braitman LE, Trock BJ, Schultz D, et al. Model predicting survival in stage I melanoma based on tumor progression. J Natl Cancer Inst 1989; 81:1893-904.

2. Clemente CG, Mihm MC, Bufalino R, Zurrida S, Collini P, Cascinelli N. Prognostic value of tumor infiltrating lymphocytes in the vertical growth phase of primary cutaneous melanoma. Cancer 1996;77:1303-10.

3. Pritchard ML, Woosley JT. Comparison of two prognostic models predicting survival in patients with malignant melanoma. Hum Pathol 1995;26:1028-31.

4. Mihm MC, Clemente CG, Cascinelli N. Tumor infiltrating lymphocytes in lymph node melanoma metastases: a histopathologic prognostic indicator and an expression of local immune response. Lab Invest 1996;74:43-7.

5. Lowin B, Pieatsch MC, Tschopp J. Perforin and granzymes: crucial effector molecules in cytolytic $\mathrm{T}$ lymphocyte and natural killer cell-mediated cytotoxicity. Curr Top Microbiol Immunol 1995;198:1-24.

6. Berke G. The CTL's kiss of death. Cell 1995;81:9-12.

7. Anderson P, Nagler-Anderson C, O'Brien C, Levine H, Watkins S, Slayter HS, et al. A monoclonal antibody reactive with a $15-\mathrm{kDa}$ cytoplasmic granule-associated protein defines a subpopulation of CD8+ T lymphocytes. J Immunol 1990;144:574-82.

8. Anderson P. TIA-1: structural and functional studies on a new class of cytolytic effector molecule. Curr Top Microbiol Immunol 1995;198:131-43.

9. Tian Q, Streuli M, Saito H, Schlossman SF, Anderson P. A polyadenylate binding protein localized to the granules of cytolytic lymphocytes induces DNA fragmentation in target cells. Cell 1991;67:629-39.

10. Szymik B, Woosley JT. Further validation of the prognostic model for stage I malignant melanoma based on tumor progression. J Cutan Pathol 1993;20:50-3.

11. Elder DE, Van Belle P, Elenitsas R, Halpern Q, Gerry D. Neoplastic progression and prognosis in melanoma. Sem Cut Med Surg 1996;4:336-48.

12. Rosenberg SA, Packard BS, Aebersold PM, Solomon D, Topalian SL, Toy ST, et al. Use of tumor-infiltrating lymphocytes and interleukin-2 in the immunotherapy of patients with metastatic melanoma. N Engl J Med 1988;319:1676-80.

13. Crowley NJ, Slingluff CL, Vervaert CE, Darrow TL, Seigler HF. Inhibition of the growth of human melanoma xenografts in nude mice by human tumor-specific cytotoxic T-cells. J Surg Oncol 1990;43:67-72.

14. Sabzevari H, Reisfeld RA. Human cytotoxic T-cells suppress the growth of spontaneous melanoma metastases in SCID/hu mice. Cancer Res 1993;53:4933-7.

15. Leong SPL, Zhou Y-M, Granberry ME, Wang TF, Grogan TM, Spier C, et al. Generation of cytotoxic effector cells against human melanoma. Cancer Immunol Immunother 1995;40: 397-409.

16. Schiltz PM, Beutel LD, Nayak SK, Dillman RO. Characterization of tumor-infiltrating lymphocytes derived from human tumors for use as adoptive immunotherapy of cancer. J Immunother 1997;20:377-86.

17. Reali UM, Martini L, Borgognoni L, Semino C, Pietra G, Chiarugi C, et al. Infusion of in vitro expanded tumorinfiltrating lymphocytes and recombinant interleukin-2 in patients with surgically resected lymph node metastases of malignant melanoma. Melanoma Res 1998;8:77-82. 